\title{
DYNAMICS OF REVERSE SACCADES OF READING TEXTS AND ITS CONNECTION WITH THE PECULIARITIES OF EYE MOVEMENT
}

\author{
Rostislav V. Belyaev, Vladimir V. Kolesov \\ Kotel'nikov Institute of Radioengineering and Electronics of RAS, http://www.cplire.ru \\ Moscow 125009, Russian Federation
}

\section{Galina Ya. Menshikova}

Lomonosov Moscow State University, http://www.psy.msu.ru

Moscow 125009, Russian Federation

Alexander M. Popov, Viktor I. Ryabenkov
MIREA-Russian Technical University, http: //www. mirea.ru
Moscow119454, Russian Federation
belyaev@cplire.ru, kvv@cplire.ru, menshikova@psy.msu.ru, ryabenkov@mirea.ru, popov@mirea.ru

Abstract. The study of the work of the human visual apparatus is of interest both for the purpose of an in-depth understanding of the vision mechanisms and for the possibility of penetrating the cognitive links of the work of the vision canal with the process of the brain. The dynamics of eye movement in the perception of graphic information and, in particular, when reading different texts makes it possible for investigating the individual physiological parameters of the human visual apparatus. In the work, specific features of the eye movement on reverse saccades related to reading the text and rapid translation of the view from one line to another were studied. This process is not connected with the cognitive work of the brain and, apparently, is determined only by the physiological features of the visual apparatus.

Keywords: eye tracking technology, information perception, text reading, dynamic eye model, involuntary eye movements, microsaccades, saccades

UDC 004.932.2; 159.931

Bibliography - 10 references

Received 02.06.2018

RENSIT, 2018, 10(1)117-127

DOI: $10.17725 /$ rensit.2018.10.117

\section{Contents}

1. INTRODUCTION (117)

2. Apparatus and methods of Research (118)

3. ANALysis of Eye Motion ON REVERSE SACCADES (120)

4. Dynamic eye model (123)

5. SPECtral ANALYSIS OF EYE MOtion ON REVERSE SACCADES (124)

6. Conclusion (126)

REFERENCES (126)

\section{INTRODUCTION}

Operation channel of visual perception of men also as other living creatures provide the impotent information channel playing critical role in determining of their existence in environmental world. And this is a reason while with investigation of this channel are connected activity of many men of science. And with functional of this channel is there are connected efforts of many men of science with problem of cognitive activity of men. In the row with other possible means of such kind of this investigation is possibility to follow for moving eyes of looking men in process of such type of activity and perception an information about nearby world. Interest to this questions lead to creation different technical means and special apparatus permitting to follow, registered and investigated processes of man's eyes moving and modern progress in computer technology open wide possibility of study and research of different variety manifestation of working apparatus of vision and its functions. There are made variety of apparatus for registration of eyes moving in process of men activity and purposeful its applications for study of visual perception [1]. 


\section{APPARATUS AND METHODS OF RESEARCH}

Principle of functioning of such type assembly which was used in process of investigation represented in this material.

Moving of eyes were registered with help of special computer issue iView XTM $\backslash$ High $\backslash$ speed 1250 German firm SMI MbH (resolution $<0.01^{\circ}$, frequency of discretization $1250 \mathrm{~Hz}$ ). Operation of this apparatus is based on next: head of observer taking part in experiment set on defined distance from display of monitor $(80 \mathrm{~cm})$ on which there are represented needed image strictly fixing for time of experiment. Source of IR radiation which very strictly reflects by all parts of eyes surface but not on pupil is fixing relatively to the face of observer. So that spot of IR radiation is overlay the region of pupil. In this conditions level of power IR radiation is adequate for experiment but not reach dose of danger for men' eye. Parameters of IR image of eyes were registered by video camera and fixing in data file [2]. Calibration was made on the base of special system of points on display of monitor in conditions of fixing position of and series stay view of observer on this points. And on special algorithm there were fined an connection between place of view on the display and position of observer's pupil. Additively there used information about position of blink from lamp of project IR on the surface of observers eye. Apparatus is working in regime of dark eye. As result of working such system it is convenient to say about following for view on the display but not of moving eye. Out of some possible type of moving eye different in time and in space there were saccade, fixation and patterns of eyes moving forming out of series of sampling out of fixations and saccades. Trajectory of mapping moving of view made by connection of points with series in time coordinates defined by frequency of sampling through equal intervals of state in time moving of men's eyes when he was made something.

It is known that in experiment with registration view moving on display of monitor on which created realization of one or other image basely there is registry two type of moving of observers view-micro saccades fixations and relatively short moving - short change of direction displace and relatively long having at an average approximately in one direction saccade.

One of the type of activities of men are connected with moving of eyes is reading texts. Out of many objects in experiments with observing images on display of monitor is selected by its feature reading of texts which as a rule state out of some number lines. They have specivity by appearance of two type of saccade - short one (in interval between words) and long one (from end of one line to the start of next one) The last saccades it is more convenient to name as return saccades (RS). Analysis shows that in RS commonly there are no fixations and on them must more significantly appears individual features of moving eyes of observers [3, 4].

One specificity of this point of view that in process of reading text every one with transfer from one line to the next line of text it must have place displacement of view for men reading one line of text to the next line of text and on this transfer there is not see appearance of other type of displacement of view such as fixation. That means displacement of view has more free character of moving but must be defined by need transfer on the start of the next new line. In this conditions may appears other individual physical features of a men's eye - possible dimension of field of view concrete man that means that is his times and space characteristics. Experiments approves this conception. On this process definite impact certainly may also effect length of lines and distance between lines [5, 6].

In represented here results of experiments take part 8 observers to which there were represented for reading 12 different texts with equal numbers of lines and accordingly return saccades. Time of reading every text was fixed. Text were not known beforehand. Participants were students and professors of Lomonosov Moscow State University. They did not have any special selection and so was realized some inevitable dispersion of individual features. Full number of received material is rather big and here used only part of it for illustration.

Interest of using texts reading connected with fact that this event distinguished from ordinary experiments with looking at some images. As a rule in last case trajectory of moving eyes defined sufficiently arbitrary and connected with given problem. In case of reading texts there are given 
rather definite way to read text and so transfer from the end of one line to the start of next one. And just here on this transfer may appear individual features of every observers apparatus of vision [7].

Typical realization trajectory of moving observers view while reading text presented on Fig. 1. Here are seen clearly basic features of moving view on display of monitor on which there mapping readable text. Part of trajectory along readable line connect two type of local moving - relatively short parts with small length amplitude with sharply changes of direction of moving (this part called - fixation) [8]. And between them are more plane and to some extant having more length so called saccades. Last one called little saccades. And trajectory transfer on next line of text called realization return saccades (RS).

The sequence data of coordinate $X$ and $Y$ realization of moving view on display of monitor realized sampling with frequency $F_{m}=1250 \mathrm{~Hz}$ and correspond to increasing of order number $n$ forming by apparatus of vision [9]. Here as on other Figures there are using next notation: letters - observers for

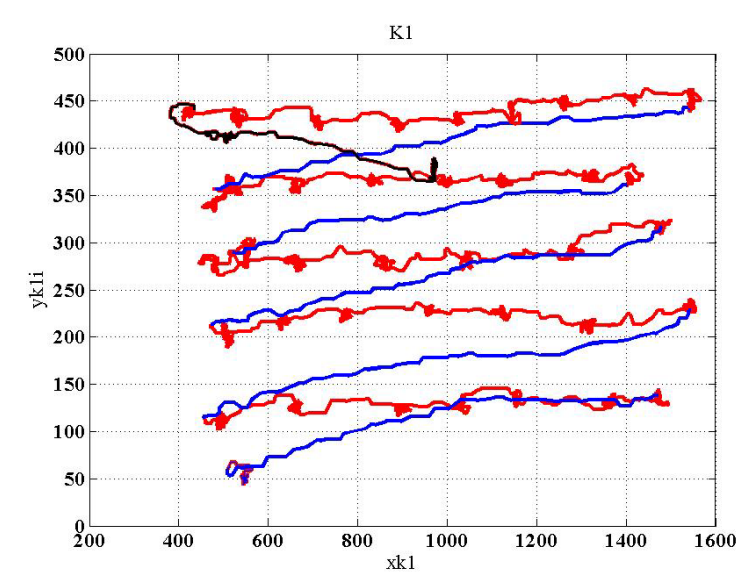

Fig. 1. Realization k1.

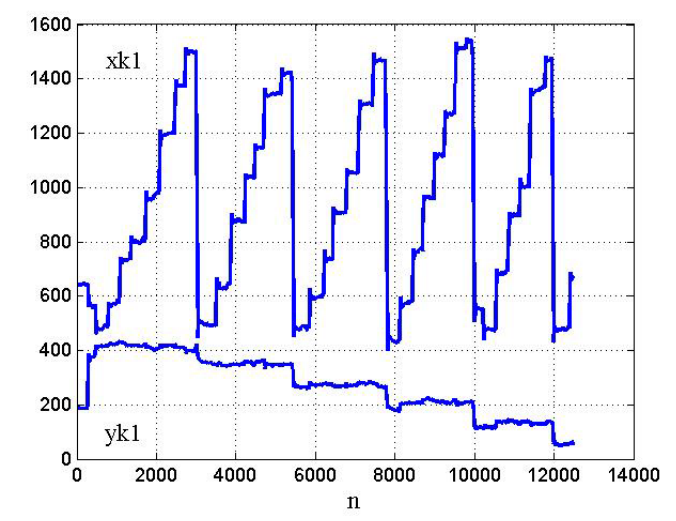

Fig. $1 a$. Realizations xk1 and yke1. example A or $\mathrm{K}$, and number 1 or 2 or other digit number of readable text.

For comparison in parallel represented realization for 2 observers $\left(k_{1}\right.$ and $\left.a_{1}\right)$ reading one and the same texts where digit nominate number of text Fig. 1, Realization $k_{1}$ and Fig. 2, Realization $a_{i}$; Fig. 3, Realization $k_{2}$ and Fig. 4, Realization $a_{2}$.

For the convenience of comparing the trajectories of eye movements of different observers, separate projections along the $X$ and $Y$ coordinates are also given. In the figures, the first inclined part of the movement (the black line of the line of sight displacement) with relative initial coordinates $X \sim$ $320, Y \sim 1000$ corresponds to the initial position of the subject's view prior to the start reading, and its end is observed near the beginning of the first line of the text (Fig. 1a and Fig. 2a).

In Fig. 1a and Fig. 2a shows the implementations $x k 1$ and $y k 1$ and the implementations $x a 1$ and $y a 1$. This representation is very convenient, since it demonstrates simultaneously the ratio of the rates of displacements. These curves show the temporal and quantitative relationships between the displacements along the $X$ and $Y$ coordinates. They clearly show

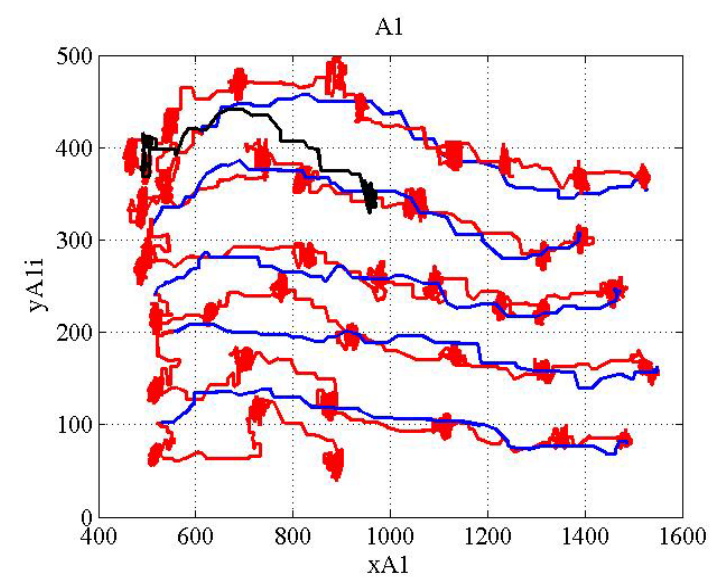

Fig. 2. Realization a1.

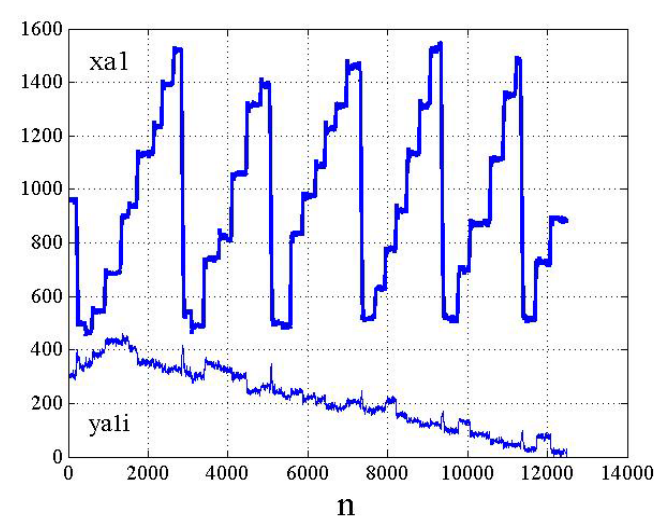

Fig. $2 a$. Prealizations xa1 and ya1. 


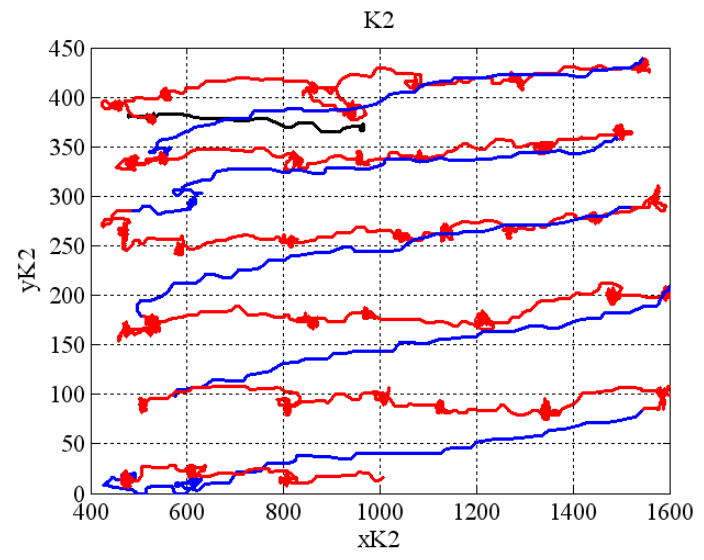

Fig. 3. Realization k.2.

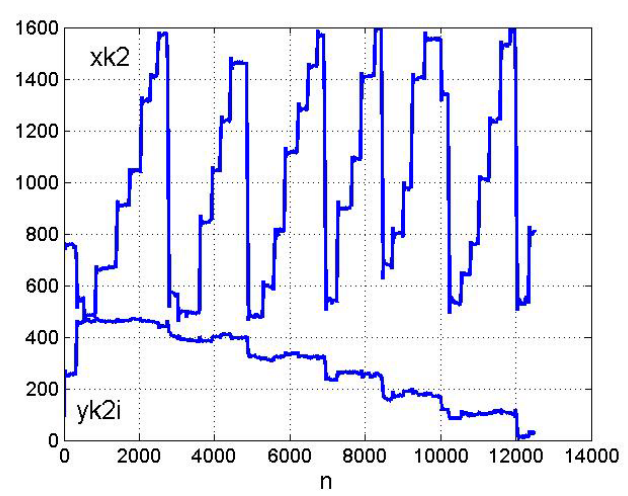

Fig. 3a. Realizations $x k 2$ and $y / k 2$.

that the movement on the reverse saccades occurs unevenly in time, and much faster than in the direct reading area.

\section{ANALYSIS OF EYE MOVEMENT}

On the base of experiments with observers there were selected two extreme opportunity of realization which very likely most impressively difference on received results. One of them (observer $\mathrm{K}$ ) demonstrated the most flat motion on return saccades out of all other observers. And other observer (A) on opposite way demonstrated one of the most uneven motion on return saccades. All others (6) observers taking part in investigations by characters of motion on return saccades approximately may be set in middle group between first two. Adequate full realization for all process of reading without elimination of return saccades for indicated before for two observers $\mathrm{A}$ and $\mathrm{K}$ are given on start Fig. 1a and 2a.

On the Figure 1 and Figure 2 line of black color indicated start of motion to start point of text, trajectory of red color indicated to parts of start

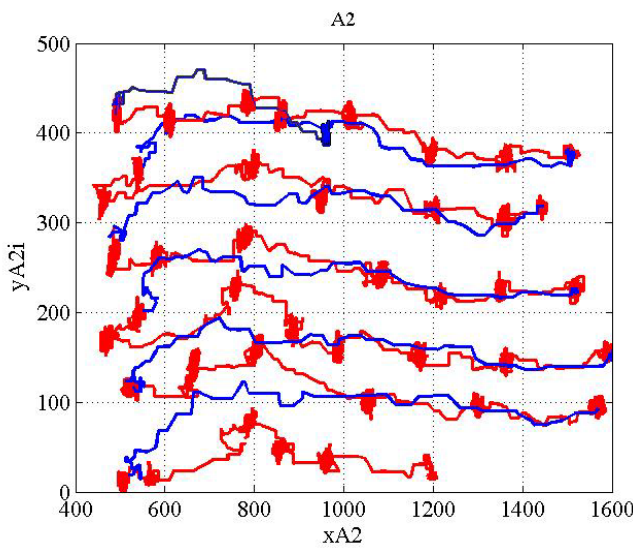

Fig. 4. Realization a2.

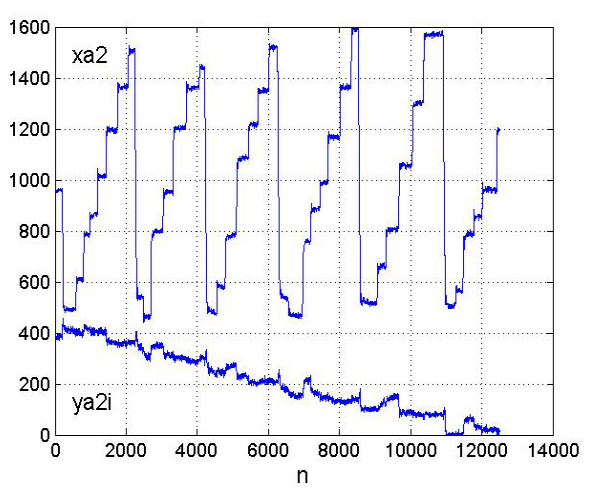

Рис. 4a. Realizations xa2 and $y a 2$.

reading text and trajectory blue one indicated to return saccade.

On the Figure $1 \mathrm{a}$ and Figure $2 \mathrm{a}$ for convenience of comparison there are given every realization of reading text by one of observers states appropriate adequate matching in time for different representation for every of coordinate $\mathrm{X}$ и $\mathrm{Y}$ in dependence of number $\mathrm{N}$ of sampling and moment of start. Shown on Fig. 1a, 2a, 3a, 4a data shows that with changing of number of in region of return saccade changing of coordinate $\mathrm{X}$ in many case considerably more then changing of coordinate $\mathrm{Y}$ and so in many cases by last changing of coordinate $\mathrm{Y}$ it is possible it to neglect. It is possible suppose that observed difference in characteristic of return saccades for different observers is connected with different field of view for everyone of them.

Further investigation with using an analysis of other type moving let us expand representation about real eyes dynamics and make belief to connect them with conception of real eyes dynamic and with individual feature of eyes of different observer $s$. 


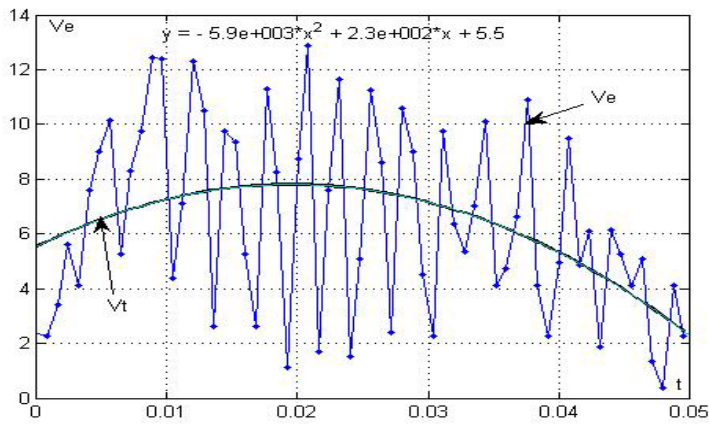

Fig. 5. Change the instantaneous movement speed of the view along the saccade.

Let us take first return saccade by using for it Fig. 2a but without using feature of return saccades. We make creation for this a dependence on time current conditions of speed moving of view along saccade $(V$ by superposing start point of counting reading on the saccade for Fig. 5. This figure clearly shows tendency of current speed moving in time: from the start speed of saccade is increasing, reach maximum value and after diminished to the end of saccade.20Let us made approximation by polynome of 2 -nd power (Fig. $5)$ and write speed in common view

$$
V_{e}(t)=P_{v}(1) t^{2}+P_{v}(2) t+P_{v}(3),
$$

where $P_{n}(n)$ are the power coefficients of the polynomial.

Representing the instantaneous velocity in this form, we obtain the dependence on the time of moving the view along the trajectory

$$
S_{t}=P_{v}(1) t^{3} / 3+P_{v}(2) t^{2} / 2+P_{v}(3) t
$$

The same dependence we may received on the base of experimental data by summing up moving on the saccade for every step of calculations and signify

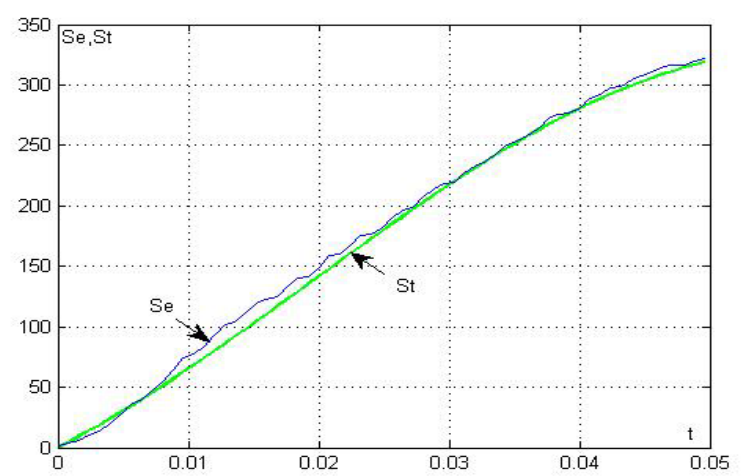

Fig. 6. Changing the length of the movement along the reverse saccade of St and Se, determined in two ways.

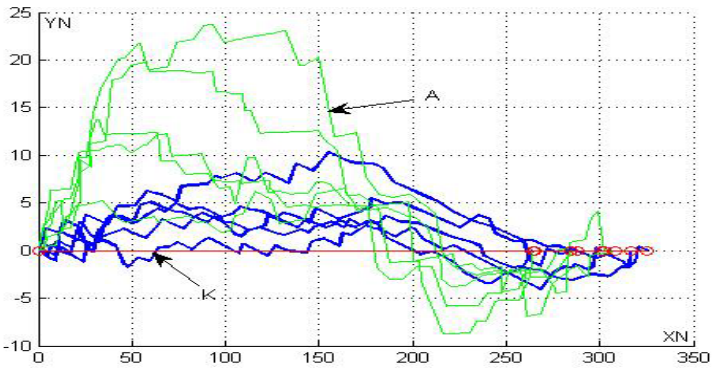

Fig. 7. Standart deviation (SD) for two observers $K$ and $A$ on reading the same text

sum value by Se. On the Fig. 6 for dependence of time $t$ there are represented $S t$ and $S e$. Coincidence is reliance with taking into account, that $S t$ received with help of approximation of experimental data of speed which for one's turn depend on calculated data experimental coordinates in which there were data errors.

In experiments take part in 2 observers $A$ and $\mathrm{K}$ read on one and the same text with nearly 5 . lines (Fig. 3 and Fig. 4).

Really true return saccades distinguished from proposed ideal right line. This distinction may be characterized for every saccade by one number - standard deviation (SD). It was summing up a squared root of deviations for every one points of real saccades from ideal line between first and last point of real saccade. This procedure is convenient to do in a new system a frame of reference. To made transfer to a new frame of reference so that line goes through start and end points of saccade goes along new axis $X$. And first point of new system superpose with start point of saccade. This procedure illustrated by Fig. 7 where for the every saccades line combine with axis X.

Calculated distinction SD and distinction relative to number of point $\mathrm{L}$ on the saccade $\mathrm{SD} / \mathrm{L}$ presented in Table 1.

Table 1

The standard deviation (SD), as well as the deviations referred to the number of points $L$ on the saccade

(SD/L) for the 5 saccades for subjects $A$ and $K$

\begin{tabular}{|l|l|l|l|l|l|}
\hline $\begin{array}{l}\text { Number of } \\
\text { saccade }\end{array}$ & 1 & 2 & 3 & 4 & 5 \\
\hline A: SD & 132.35 & 110.16 & 64.43 & 56.45 & 79.91 \\
\hline K: SD & 46.31 & 25.99 & 34.45 & 41.15 & 50.28 \\
\hline A: SD/L & 1.79 & 1.81 & 1.04 & 0.83 & 1.23 \\
\hline K: SD/L & 0.74 & 0.50 & 0.56 & 0.65 & 0.90 \\
\hline
\end{tabular}




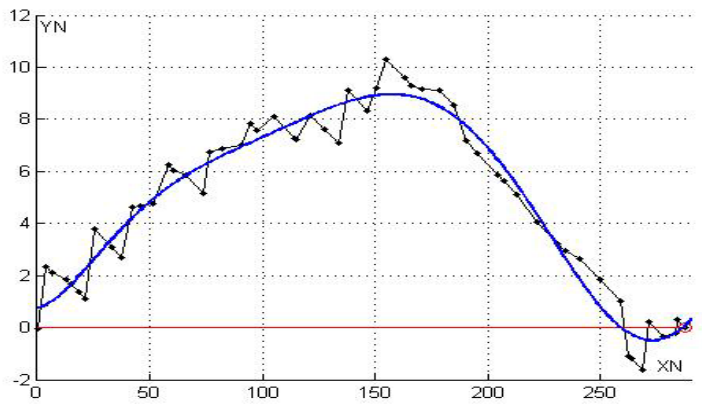

Fig. 8. Approximation by polinom 7-th power real registrate apparature trajectrory of moving view of observer $K$ on reading text 2 for saccades number 4.

It is clearly seen from the Table 1 that for observer $\mathrm{K}$ a substantially more even viewing of the implementation is characteristic, (the values of $\mathrm{SD}$ and $\mathrm{SD} / \mathrm{L}$ are significantly less than for observer A).

Out of Fig. 8 and data in Table 1 it is possible to draw a conclusion that behevior of view on return saccades for observer $\mathrm{K}$ is more rational from the point of view to minimize process of text-reading.

It is nesessery to pay attention to one more fact. On the Fig. 5 are shown two speeds: Ve-calculated on the base of experimental data for view and Vtapproximation those speeds by polinom of 2-th power.

Assumption that view must moving by more short type that means on line of return saccade is not fully wright. As eyes certainly see the full of text but its detail distincly differy in when their image appeared in region of eyes fovea and so ees must moving as if as "gropingly", sequenshially moving to the final aim (start of next line). So, counterpart saccade must have must be rather complex configuration but its form must be smooth and describing its curve - be differential. If change real saccade by polinom 7-th power then indicated polinom it will be logically to consider to be more nearly to real trajecory of view, which is distortion by noises of system of registration (Fig. 8).

Now real points of traking deflection out of polinom will be looked at as noise and concequently if it will make distribution for such type deflection then it must be simmetric relatively to zero and it wil described by normal law. On the Fig. 9.1 are apportionment for return saccade

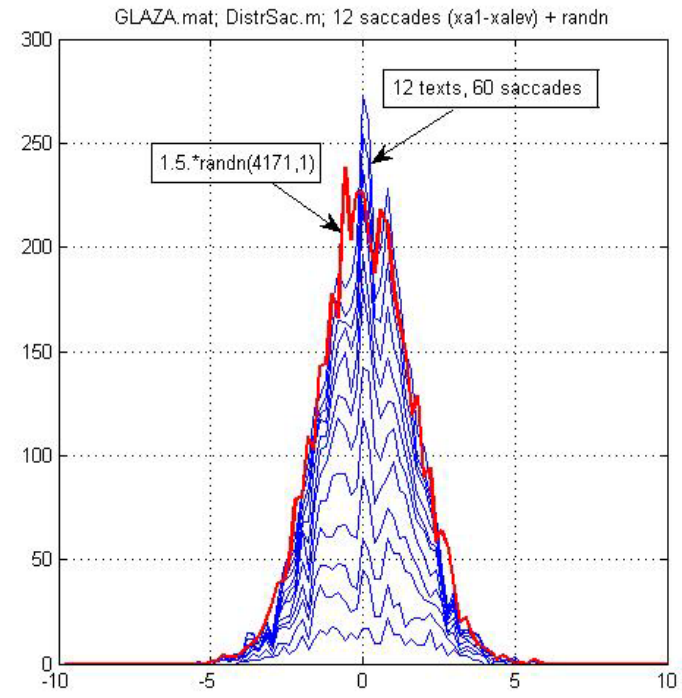

Fig. 9.1. Statistic of saccades distribution for observer $A$ when be was reading in series 12 texts. External red line of distribution represents statistic of $M A T L A B$ generator of random numbers with distribution nearly to $\sigma=1.5$.

for observer $A$ in conditions of sequentially growth of statistics (number of read-throw new texts). All number of text 12 and every one of them connect 4-5 return saccades. And so full number of sampling in distribution 4171. And total number of sampling on this Fig. 8 compose 4171. On this Figure the most "thick one" outcurve shows distribution received with the same statistics received with MATLAB genearator of random numbers with the same statistics and standart deflection $\sigma=1.5$. Coincidence of distributions is appearently. This fact let us make conclution that real level of noise in this aopparature for register coordinats of view are the

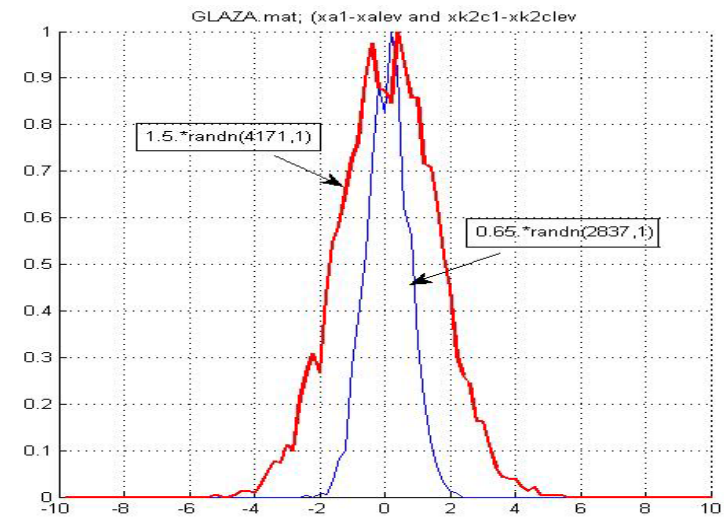

Fig. 9.2. Statistic of saccades distribution for observer $K$ when he was reading in series 12 texts. External red line of distribution represents statistic of $M A T L A B$ generator of random numbers with distribution nearly to $\sigma=0.65$. 


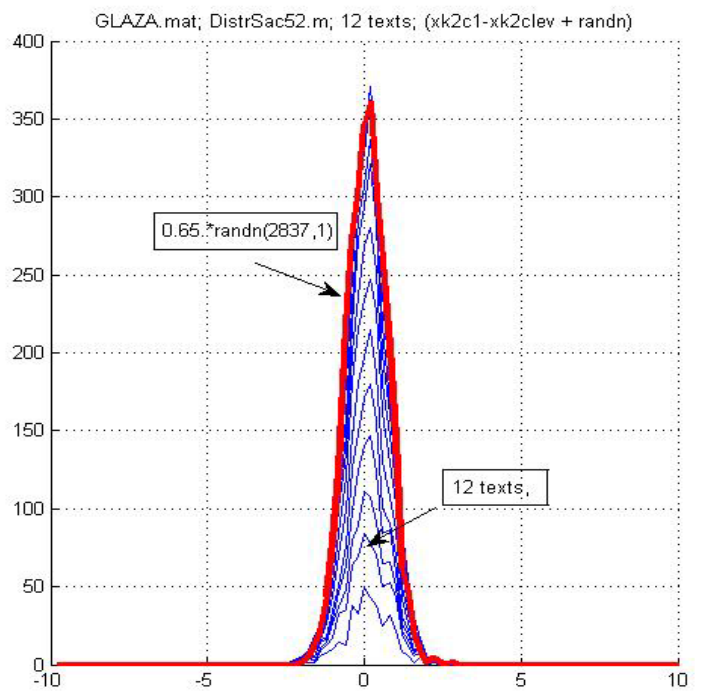

Fig. 9.3. Results for data comparison statistics of MATLAB generator of random numbers with distributions to agree with result of return saccades statistics, realized for sum of distribution for reading 12 texts by early shown different observers $A$ and $K$. External red line agree with result statistic of generators $M A T L A B$ with random numbers of distributions $\sigma=1.5$ and intrinsic blue line agree with $\sigma=0.65$.

same level of value as $\sigma$. Analogical procedure for observer $\mathrm{K}$ gives value $\sigma=0.65$ (Fig. 9.2). These facts directly adressed that error of evaluation is depend on concret individual eyes. On Fig. 9.3 there are given appresiation resulting statisics for generator of random numbers of MATLAB with disributiions according to reverd succades approved to summer distribution on reading 12 texts by shown beforhand for different obsevers recording summer distribution by early shown different observers. Analogycal procedure fulfield for observer $\mathrm{K}$ show gives value $\sigma=0.65$ that also indicate that error of evaluation eyes movements depend also on concret eyes (Fig. 9.1)

\section{DYNAMIC EYE MODEL}

Based on the measured characteristics, it is possible to construct a simple dynamic model of the eye.

Out of (1) it may be received dependence of the eyes acceleration while moving on return saccade $-w_{i}$

$$
W_{\mathrm{t}}=2 P_{\nu}(1) t+P_{\nu}(2) \text {. }
$$

Here $L=0.8 m-$ is distance out of eyes to screen and namely angle acceleration is most impotent parameter of eyes revolt kinematics. Let us take equation of eyes moving

$$
I \varepsilon_{\mathrm{t}}=M+\mathrm{M}_{\mathrm{s}}
$$

where $I$ is the moment of inertia of the eye relatively axis of revolution $\varepsilon_{\mathrm{t}}, M$ is the moment of forces acting on the eye, $M_{\mathrm{s}}$ is the moment of all resistance forces acting on eyes, which, apparently, we must be take as sphere with radius $R \sim 1 \mathrm{~cm}$ density of which is nearly of water $\varrho=10^{3} \mathrm{~kg} / \mathrm{m}$ and if axis of revolution goes through geometric centre of eyes the moment of inertia will be equal

$$
I=(8 / 15) \pi \varrho R^{5}=1.7 \cdot 10^{-7} \mathrm{~kg} / \mathrm{m}^{2} \text {, }
$$

what probably is minimal value, since any movement of revolution axis of centre of eye only will make more this evaluation. Moment of force effect upon eyes equal to product of force on arm and this arm will be maximum if the force will be directed on at a tangent to spheric retina of eye. In this case $M=F \cdot R$ where $F$ - result of all forces taking part in revolution of eye. Taking into consideration connection between angle and linear acceleration of view and (4) we will have resultant force

$$
F=(8 / 15) \pi \varrho R^{4} w_{t} / L \text {. }
$$

Here $L=0.8 \mathrm{~m}$ is the distance from the eye to the screen with the text.

That means resultant force is quota to linear acceleration of view. This conclusion let us make evaluation of maximal value of force. As maximal value of acceleration equal to $P_{\nu}(2)$ that means it is equal $250 \mathrm{~m} / \mathrm{s}^{2}$ Take its value of max acceleration

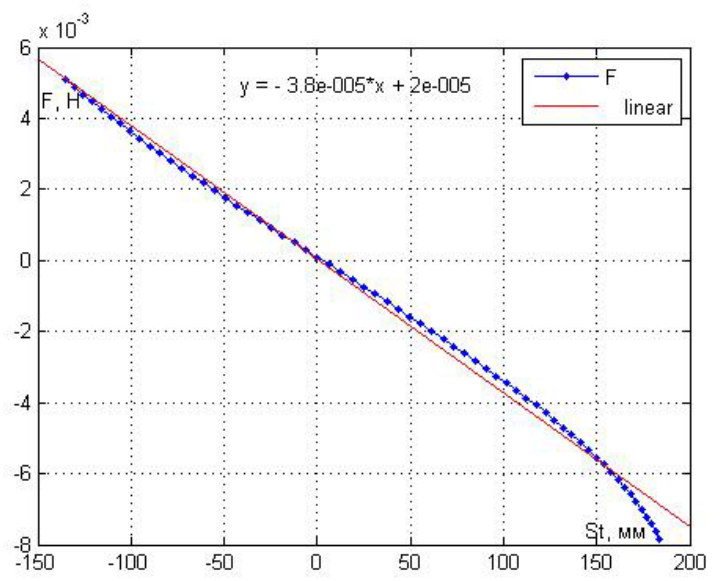

Fig. 10. Connection between resulting "quasy-elastic" force and displacement out of equilibrium position for approximated spherical model of eye. 
and parameters of eye given earlier for max value of force we will have value order $6 \mathrm{mN}$.

Interestingly such type of dependence of this force at displacement out of equilibrium position It may be taken as centre of image (text). In this position muscles are relax and force is equal to zero. For making such kind of dependence we have all needed: we know dependence as of acceleration (6) and of displacement of eye (2) on time. Such type of curve is represented on Fig. $\mathbf{1 0}$ where also it is represented approximation of this dependence by linear law.

As you can see there is rather commendable coincidence and force revolting eye on moving along return saccade have all features of "quasielastic" force. Such type of features have force acting on weight from the spring. At like action of such type of force in system there are appeared either oscillations either system smoothly return to equilibrium position (at condition of large resistance). Comparison of forces acting on eye like spring apparently is not correct as such forces are "guided" but their "quasy-elastic" is intriguing and must be mentioned.

It seems that in eyes revolting take part not one muscle but acting together they are equivalent to one spring and nearly definitely obey to Hookes' law. And so $M$ moment of force revolting eye may be described as

$$
M=k \times R=k L R \varphi \text {, }
$$

where $\varphi$ is the angle of turn out of equilibrium position , $k$ - "coefficient of inflexibility" of spring may be evaluated out of Fig. 10 and it could have value in order $4 \cdot 10^{-2} \mathrm{~N} / \mathrm{m}$. Describe equation of moving (4)

$$
\frac{d^{2} \varphi}{d t^{2}}+\frac{k L R}{I} \varphi=0
$$

Expression (8) represent as equation of characteristic oscillation and coefficient before the variable $\varphi$ in first power - is square of characteristic oscillation $\omega_{0}$. Let us evaluate this frequency $\omega_{0}$, taking values of variables shown early

$$
\omega_{0}=\sqrt{\frac{k L R}{I}} \approx 41 \mathrm{rad} / \mathrm{s} \approx 6.5 \mathrm{~Hz} .
$$

Most probably this frequency doesn't appeared as we have deal not with classical spring and neglect by resistive forces.

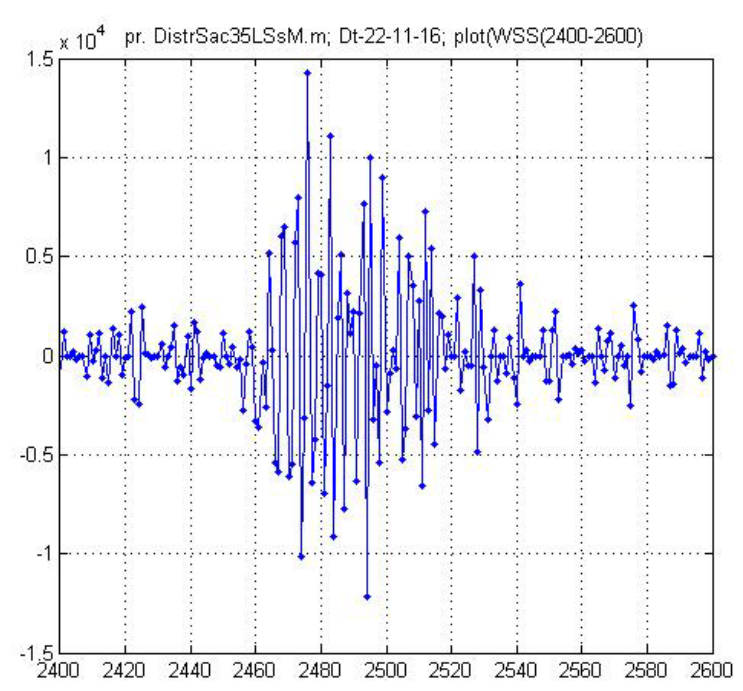

Fig. 11. Dependence of the horizontal component of acceleration on time one of the $\mathrm{RS}$

Let's look at the nature of the look at the RS. In Fig. 11 shows the dependence of the horizontal component of acceleration on time in the vicinity of one of the RS. The instantaneous value of the acceleration is calculated as the ratio of the increment of the horizontal velocity component to the time interval for which it occurred $T=1 / f$, where $f=1250 \mathrm{~Hz}$ is the detection frequency of the viewing position. It should be noted that the vertical coordinate of the view of the RS is practically unchanged, and for this reason the corresponding components of speed and acceleration can be ignored.

The figure shows that the value of acceleration on the RS is much larger than on neighboring "fixations" and, in addition, there is a periodic component, the frequency estimate of which gives a value of about $300 \mathrm{~Hz}$.

\section{SPECTRAL ANALYSIS OF EYE MOTION ON REVERSE SACCADES}

As it was checked previously Return Saccades (RS) in consequence of characteristic for their fast movements have relative small time-length (number of sampling) in comparatively with movement in process of direct text reading. In search of individual features of RS for every one of eyes moving on observer on various text-reading it was undertaking investigation of spectrum characteristics of such type moving with using algorithm of fast Fourier transform (FFT). There were used next parameters of FFT: a basic parameter NS $=32$ with natural 
frequency of sampling $F_{\mathrm{m}}=1250 \mathrm{~Hz}$ in process of offset on next RS after every one read-through every one line in text. For every one observer process of FFT was performed sequentially for every text. It was appeared that best performing the analysis from the point of view of get spectrum by using mean value of length of calculation deviations from this mean value. And to those values realized process of FFT. As there used only linear operations on very one step of this process it means that it may be confident that if the interesting for us periodic really exist that it will not disappeared and it could not appeared as result of linear process of computations.

There are expected specific feature for RS possibly may be connected with physiological features and they will ot masking by such situation as possibility view not only in region of some fovea information content of some reading text (as here really there isn't any process of reading).

It is possible that here may appeared such type of situation that it could see not only in region of fovea himself but also to see with a more little resolution but in more wide region. That must made it output on start of next line of text and it made more light to find output on the start of next text's line for its reading.

For getting presentation of features of movements on RS there are given appropriate results for the same observers $A$ and $K$ as previously known proxy and with adding with an observer $\mathrm{N}$. The last one is more nearly to mean of distribution for all people taking part in experiments. But a last one

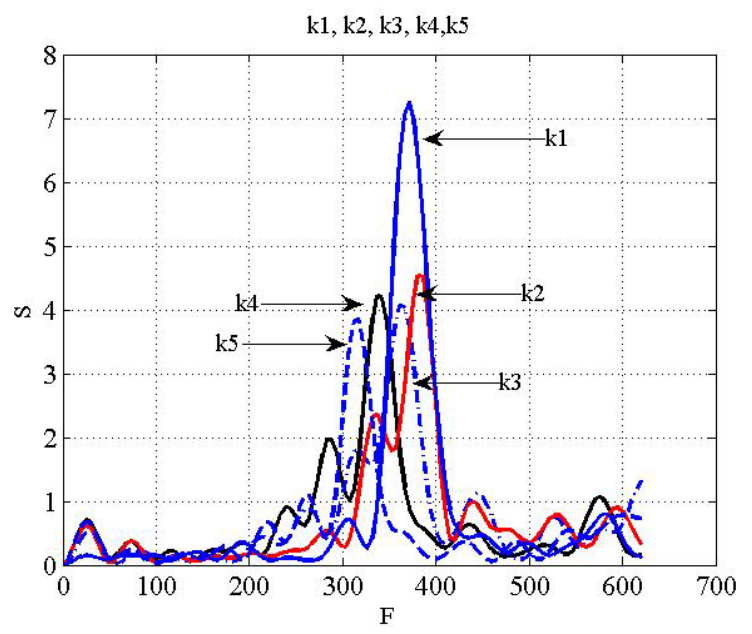

Fig 12. Frequency spectrum of eye movements on reverse saccades for observer $K$ when reading texts 1, 2, 3, 4, 5 .

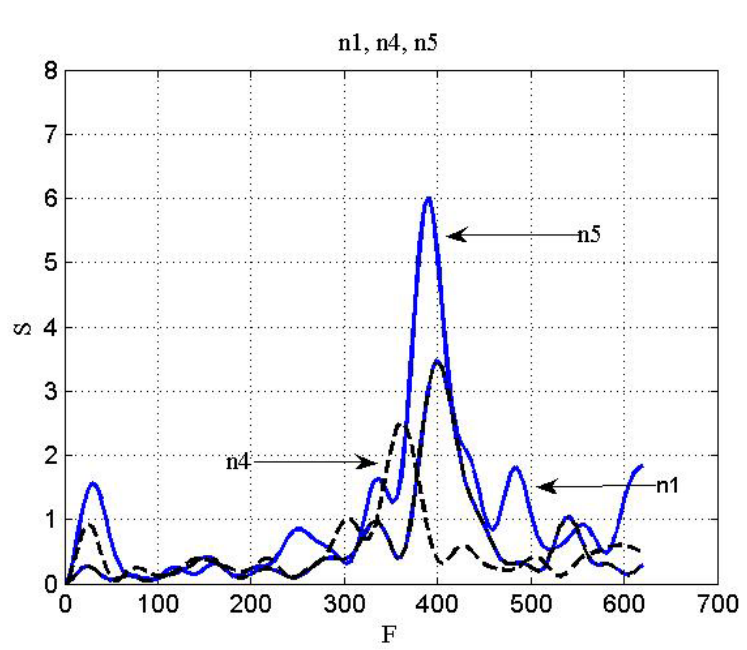

Fig. 13. Frequency spectrum of eye movements on reverse saccades for observer $N$ when reading texts $1,4,5$.

observer $\mathrm{N}$ is to some extent more nearly to observer $\mathrm{K}$ by characteristics s of its spectrum. On the Fig. 12 there are diagram of spectrum for observer $\mathrm{K}$ on reading texts $1,2,3,4$ and 5 . Here on the axis $\mathrm{X}$ is frequency in $\mathrm{Hz}$ and on axis $\mathrm{Y}$ is amplitude $\mathrm{S}$ in relative numbers. It may see that RS for different texts have different values of oscillations but all of them stay in region of $300-400 \mathrm{~Hz}$. Similar situation realized for other observer $\mathrm{N}$ when he looking throw texts 1, 4 and 5 (Fig. 13).

Other type features have spectrum of realization for observer A (see spectrum on diagrams of Fig. $14 a$ and 14b). In this case traceable spectrum are distributed on analyzed wide frequency band. When at some times it appears more on values spectrum spikes with relatively to average spectrum level. In
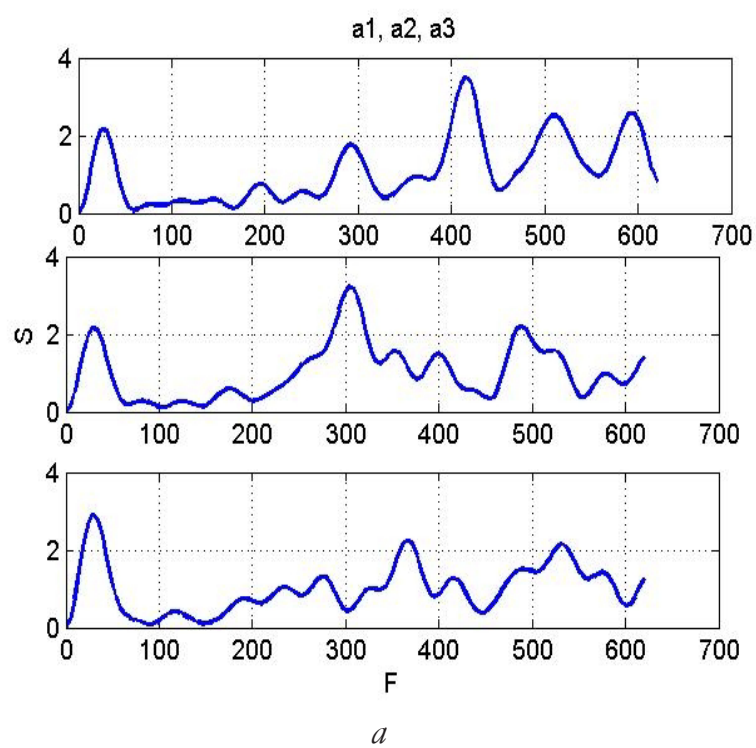

Fig 14 a. Frequency spectrum of eye movements on reverse saccades for the observer $N$ when reading texts 1, 2, 3, 4, 5, 6 . 

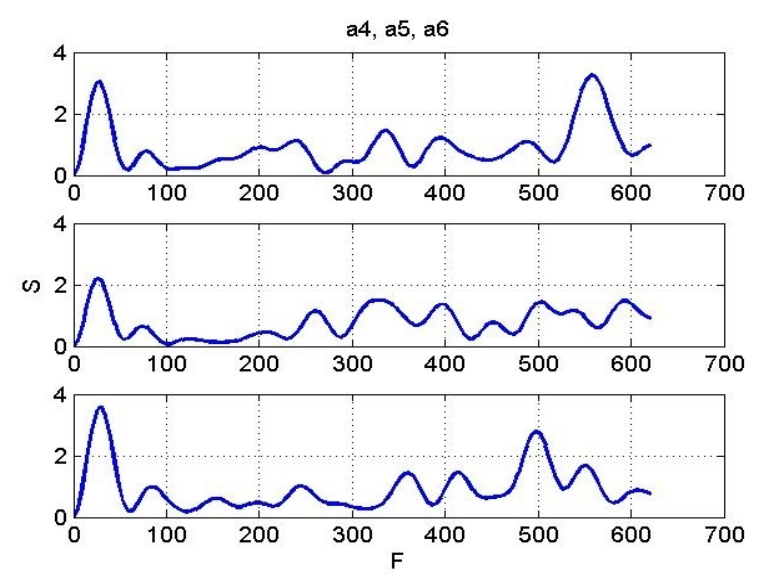

$b$

Fig 14b. Frequency spectrum of eye movements on reverse saccades for the observer $N$ when reading texts 1, 2, 3, 4, 5, 6 .

addition it stably appears some oscillations on very small frequency nearly of $50 \mathrm{~Hz}$.

In this situation first of all as it possible to see on diagrams of spectrum it was appeared not text specific but characteristics of concrete observer. It is possible to see on diagrams one of spectrum oscillations with frequency nearly $550 \mathrm{~Hz}$.

It is interesting to pay attention to the facts that process of text-reading the moving of views on return saccade it takes place as necessary element of all process text-reading. There is not simple displacement but realized moving with features of oscillations. It is possible that this oscillations are images of feature of some observers (as reading men). In this circumstances there are appeared features connected with visual perception system of men.

\section{CONCLUSION}

There were defined feature distributions of momentary values of selection on lengths of return saccade for some observers (as reading men). In this circumstances there are appeared features connected with visual perception system of men.

It was shown that while reading the same text resulting sum of distributions received for observers is approaching to normal distribution.

There are given approximate evaluation of turning eyes cinematic which is connected with eyes working as organ receiving information about nearby word with aim of comparison possibly proper frequencies of eyes moving.

Further investigations with connection analysis of other type eyes moving let us expand representation about real eyes dynamics and make sure possibility to connect them with individual features of observers eyes, for example, with relative dimension of fovea, for example, for eyes of different observers [10].

\section{ACKNOWLEDGEMENT}

The work was supported by the Russian Foundation for Basic Research, project 18-013-01087.

\section{REFERENCES}

1. Barabanshchikov VA, Zhegallo AV. Methods of recording eye movements: theory and practice. Psychological science and education (electronic journal), 2010, 5:240-254; www.psiedu.ru, ISSN: 2074-5885.

2. Guestrin ED, Eizenman M. General Theory of Remote Gase Estimation Using the Pupil Center and Corneal Reflections. IEEE Transitions on biomedical engineering, 2006, 53(6):1124-1133.

3. Kirpichnikov AP. Video eye processor: micromotion as a factor of accommodation and video processing. Materials $12 \mathrm{int}$. conference "Digital signal processing and its application" - DSPA-2010, Moscow, 2010, p.170-172.

4. Salvucci DD, Goldberg JH. Identifying fixations and saccades in eye-tracking protocols. Proceedings of the Eye Tracking Research and Applications Symposium, New York, ACM Press, 2000, pp. 71-78.

5. Belyaev RV, KolesovVV, Menshikova GYa, Popov AM, Ryabenkov VI. Analysis of micromotion trajectories of eyes by fractal dispersion. Theses of the 7th International Conference on Cognitive Science, Svetlogorsk-2016, Moscow, Institute of Psychology RAS Publ., 2016:144-146.

6. Menshikova GYa, Belyaev RV, Kolesov VV, Ryabenkov VI. The evaluation of individual differences using fractal analysis of scanpaths. Abstract Book of the 18th Europien Conference on Eye Movements (ECEM), 16-21 Aug. 2015, Vienna, Austria; http://www.jemr.org/online/8/4/1.

7. Lamminpya AM, Moiseenkova GA, Vakhrameeva OA, Sukhini MV, Shelepin YuE. Influence of the geometry of the fovea on the characteristics of the eye movement. Theses of the 7 th International Conference on Cognitive Science, Svetlogorsk-2016. Moscow, Institute of Psychology RAS Publ., 2016:379-380.

8. Nistrom M, Holmquist $K$. An Adaptive Algorithm for Fixation, Saccade and Glissade 
Detection in Eye Tracking Data, Behavior Research Methods, 2010, 42(1):188-204.

9. BelyaevRV,KolesovVV,Menshikova GYa, Popov AM, Ryabenkov VI. A quantitative criterion for individual differences in eye trajectories. Radioelectronics. Nanosystems. Information Technology (RENSIT), 2015, 7(1):25-33; DOI: 10.17725/ rensit.2015.07.025.

10. Belyaev RV, Kolesov VV, Menshikova GYa, Ryabenkov VI. Study of perception features of video information in telecommunication systems. Proc. 20-th Int. Crimean Conference "Microwave\&Telecommunication Technology" (Crimico'2010), 13-17 Sept. 2010, Crimea, Ukraine, p .486-487. 